\title{
A Guide to Laser-Induced Fluorescencè Diagnostics in Plasmas
}

\author{
T.G.M. Freegarde and G. Hancock \\ Physical and Theoretical Chemistry Laboratory, Oxford University, South Parks Road, Oxford OX1 $3 Q Z$, \\ U.K.
}

\begin{abstract}
A brief guide is presented for the use of laser induced fluorescence (LIF) as a diagnostic for reactive species in plasmas. The technique can determine concentrations, energies, and kinetics of atoms, radicals and ions, and can also be used to measure the fields which influence the motion of the charged particles. Measurements can be made in a spatially selective fashion within the plasma reactor, and in a way which is non-invasive. The review gives examples of all these types of measurements, with emphasis on how quantitative information can be extracted from the LIF signals. Guides are given to the limits of species detectivity, and to the pitfalls which need to be avoided if the technique is to be used reliably.
\end{abstract}

\section{INTRODUCTION}

An understanding of the physics and chemistry of plasmas requires information on identities, concentrations and energies of the species present - atoms, ions, electrons, radicals, molecules. This information needs to be quantitative, spatially resolved with respect to the active area of the plasma, and needs to be carried out in a fashion that does not disturb the plasma. If the plasma can be modulated then time resolution (on a scale which may run from seconds to ns) will provide additional information. No single technique can achieve all of these requirements, and a list of the various ways in which information of this kind has been gathered would probably encompass all techniques used in species diagnostics.

Plasmas glow, and much can be learned from observations of the wavelength resolved emission [1]. Emission reveals properties of the excited species, and is generally used in the visible and UV regions to observe electronically excited quantum states. Emission of course does not reveal the behaviour of the ground states of the species, which in most plasmas (and certainly in the low temperature plasmas used for materials processing) are in concentrations orders of magnitude higher than those of the excited states, and which are generally the major species responsible for the technologically important phenomena such as plasma etching, deposition or ion implantation. All ground state atoms, molecules and ions (with the exception of $\mathrm{H}^{+}$) can absorb light in transitions between eigenstates, and hence are detectable by this effect. Measurement of the absorption (the fraction of the incident light absorbed by the species) gives the concentration of the absorber directly, and is an extremely general technique, but suffers two drawbacks. First, the absorption is necessarily measured over the whole of the path of the interrogating light beam, and thus may mask spatial inhomogeneities. This can be overcome by viewing different paths through the plasma and using suitable inversion techniques to reconstruct the spatial variations in species concentrations. The second disadvantage is one of detectivity. Here we need to be quantitative: the limits of measurements of absorption depend crucially upon the noise characteristics of the source and detector, and the time interval over which the measurements are averaged. Some of the most careful estimates of this have come from experiments (generally not carried out in plasmas, but applicable to them) which use diode lasers as the light source. An absorption of $10^{-6}$ over a $1 \mathrm{~s}$ time period is a quoted detectivity limit with a signal to noise (S/N) ratio of unity [2], and to translate this into practical detectivity terms we consider a specific example. The 
quantum state resolved absorption features in the $\mathrm{OH}$ radical in its $\mathrm{A}^{2} \Sigma^{+}-\mathrm{X}^{2} \Pi$ transition near $308 \mathrm{~nm}$ have cross sections of the order of $5 \times 10^{-16} \mathrm{~cm}^{2}$ [3]. In a plasma of length $10 \mathrm{~cm}$, an absorption of $10^{-6}$ would be achieved by a concentration of $2 \times 10^{8} \mathrm{~cm}^{-3}$, a very respectable detectivity limit. However we reiterate that this is a limit, achieved with a well characterised laser source, and measured over a 1s time period with unity $\mathrm{S} / \mathrm{N}$. Higher measurement periods increase the problems of drift in the experimental parameters. In practice such limits would be hard to achieve in a noisy plasma environment, and a couple of orders of magnitude leeway in the absorption measurements should be allowed. We note for example that an absorption of $2 \times 10^{-4}$ has been suggested as a limit for absorption measurements of the $\mathrm{CH}_{3}$ radical with a Xe arc lamp as an excitation source near $216 \mathrm{~nm}$ [4].

Absorption measures the loss of photons, but makes no statement about their fate. Any absorption process will be accompanied by emission (fluorescence) from the excited state, and if the fluorescence quantum yield is "reasonable" (we consider what is meant by this later) then the emission can be used as a marker for the excited species, and hence for the species in the original lower quantum level. Any light source of the correct frequency can induce fluorescence, and we consider the use of a laser to do so. Fluorescence has an immediate advantage over absorption in that it is not a relative technique - in an ideal case the fluorescence is observed against a zero background. Ideal cases must give way to pragmatism, and we discuss in this review the application of laser induced fluorescence (LIF) to the noisy environment of a plasma. We first note the requirement of a "reasonable" fluorescence quantum yield. The ideal value of unity can be reduced by quenching, but this does not generally cause a major detectivity problem for low pressure ( $<100$ Torr) plasmas. What are far more troublesome are the unimolecular decay processes which can compete with fluorescence, and, for the vast majority of electronically excited polyatomic species, processes such as dissociation or internal conversion render LIF impractical. Our example of the $\mathrm{CH}_{3}$ radical considered above is a case in point - the absorption step at $216 \mathrm{~nm}$ is followed by overwhelming predissociationand essentially no useful fluorescence.

Table 1: Laser armoury. Characteristics of typical laser systems available for laser-induced fluorescence studies of plasma species. Powers and pulse energies are of course wavelength and system dependent, and the list is not intended to be exhaustive. The generation of harmonics below $200 \mathrm{~nm}$ is generally in gas phase media; the practical lower limit for third harmonic generation is taken to be the lithium fluoride transmission cut-off, although shorter wavelengths are achievable.

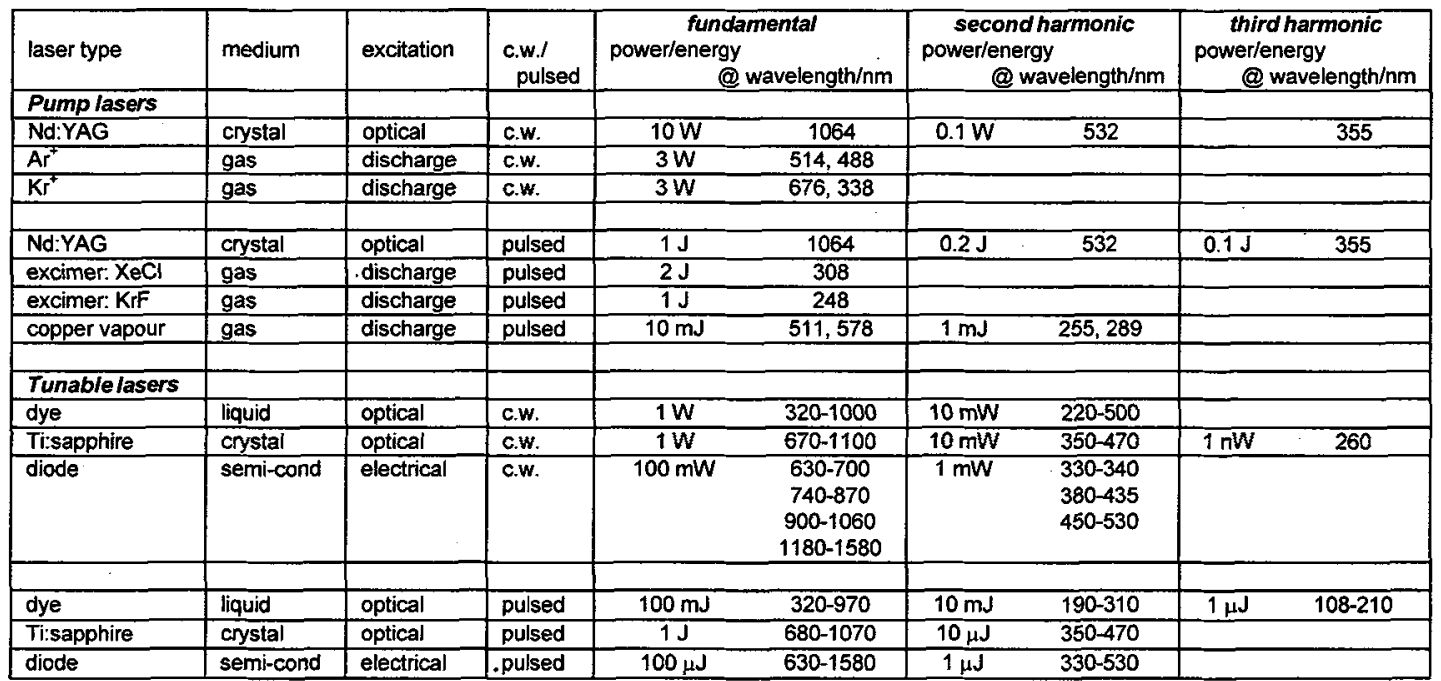

Electronic transitions which lead to observable fluorescence lie roughly in the $100-1000 \mathrm{~nm}$ region, and laser sources are available (at varying output energies and powers) at all of these wavelengths. Most experiments are carried out with tunable lasers, and the starting point is often the tunable dye laser pumped 
with the visible or u.v. fixed frequency output of a Nd:YAG or excimer system. Various frequency conversion schemes extend the output range, and examples of the laser armoury with typical output conditions are given in Table 1. Longer wavelengths take us into the excitation range of vibration-rotation transitions, and although fluorescence is still observable from these species, the relatively low Einstein A coefficients (in comparison with those at shorter wavelengths) coupled with the lower sensitivity of commonly available i.r. detectors have meant that few LIF studies of plasmas have been carried out at these longer wavelengths (although spontaneous i.r. emission can be a useful diagnostic under such conditions [5]). The characteristics of laser radiation that make the LIF technique useful are easily listed: tunability means that the absorption and hence the fluorescence is species selective, spatial coherence means that spatial selectivity within a plasma chamber is achievable with ease, intensity gives the possibility of multiphoton excitation leading to fluorescence. We shall give examples of the use of these properties in detecting atoms, radicals and ions in plasmas.

This guide seeks to be realistic. We discuss four sets of examples of the type of information that can be obtained from lasers, stressing at all times that the observations can be quantitative, but that they do have disadvantages. We first discuss the measurement of spatially resolved species concentrations in plasmas, paying particular attention to detectivity limits and to the problem of determining the absolute species concentration from the LIF signal. The next section discusses energy distributions, both translational and internal, that can be obtained by LIF, and gives an opportunity for discussion of the thorny problem of saturation by laser irradiation and how it can affect the interpretation of LIF data. We then discuss what can be learnt from temporal variations of the LIF signal, in particular commenting on the measurement of parameters such as the loss rates of species on surfaces in the plasma reactor. Finally, measurements of electric fields by LIF are briefly discussed. Several examples will be taken from the LIF measurements conducted over the past ten years in our research group.

\section{MEASUREMENT OF CONCENTRATIONS}

Figure 1 shows an example of the kind of LIF signal that can be straightforwardly obtained in plasmas [6]. Here we use a conventional RF capacitatively coupled parallel plate reactor with feedstock gases of either $\mathrm{CF}_{4}$ or $\mathrm{CHF}_{3} / \mathrm{Ar}$, at total pressures of $50 \mathrm{mTorr}$, and the species observed is the $\mathrm{CF}_{2}$ radical. $\mathrm{CF}_{2}$ has long been recognised as an important species leading to polymerisation in fluorinated hydrocarbon plasmas, and in these experiments it is excited at $234 \mathrm{~nm}$ in the strong $\mathrm{A}-\mathrm{X}$ band system with fluorescence detected off resonance (to remove problems of scattered laser light) at $248 \mathrm{~nm}$ [7]. We note several pieces of information which come from these data. First, the spatial variations of the species concentrations measured as a function of height above the RF driven electrode surface are different close to the electrode for the two feedstock gases. For an understanding of this we need to realise that the processes which form the radicals appear not to be confined to electron impact of the parent molecule: surface formation (which would give rise to an enhanced concentration close to the electrode, observed in this case for $\mathrm{CHF}_{3} / \mathrm{Ar}$ and noticeable in $\mathrm{CF}_{4}$ at higher pressures) can also play a major role [8]. Secondly, the concentrations of the radicals appear to be approximately constant in the plasma bulk (at distances above $10 \mathrm{~mm}$ from the electrode), and reflect the importance of fast diffusion ironing out any local changes in production rates. Thirdly, the data here are given on a relative scale, and this indicates one of the major problems of LIF: measurements of relative concentrations are facile, but putting these onto an absolute scale needs great care.

In principle if the characteristics of the excitation source are known, the spectroscopy of the transition is quantitatively understood, and the absolute value of the detected fluorescence can be measured, then the concentration can be determined. In practice this method is full of pitfalls, and rarely provides a convincing result. Here we first note that measurements of absolute spectral intensities of both the laser source and the fluorescence are not straightforward, and in section 3 below we shall dwell on another of the problems, that of saturation of the transition. To overcome the former difficulty, a variety of relative methods have been derived. One of these is to use a "fluorescence actinometer", a molecule whose concentration can be measured, and whose fluorescence intensity under similar conditions of laser excitation can be compared with that of the unknown species. Nitric oxide has been used in this way both for $\mathrm{CF}_{2}$ and for $\mathrm{CF}$ [9] and this 
fixes the absolute concentrations of the $\mathrm{CF}_{2}$ radicals which are given in the caption to Figure 1. We note in passing that these absolute values are determined by the balance between the radical formation and loss rates, and that both of these processes can be extremely reactor specific, because of their dependences on surface processes. The problem of saturation affecting these calibrations has been cleverly addressed [10] and we return to this below. Other relative methods have included a comparison of the LIF signal with that from Raman scattering [11], and an elegant comparison of the LIF intensities of an unknown concentration of the $\mathrm{CH}$ radical with that from the $\mathrm{N}_{2}{ }^{+}$ion, the concentration of the latter being equal to that of electrons in a nitrogen discharge, with the electron concentration measured by microwave interferometry [12]. The equality of the $\mathrm{N}_{2}{ }^{+}$ion concentration $c$ with that of the electrons in a nitrogen discharge can be used to make an estimate of $c$ by kinetic means [13]. If the discharge is extinguished, and conditions are chosen such that the ion loss process is dominantly dissociative recombination with electrons

$$
\mathrm{N}_{2}^{+}+e^{-} \rightarrow \mathrm{N}+\mathrm{N}
$$

then the rate of loss of the ions is described by a second order equation

$$
-\frac{\mathrm{d} c}{\mathrm{~d} t}=\mathrm{k}_{1} c^{2}
$$

where $k_{1}$ is the known recombination rate constant under the given experimental conditions. Analysis of the decay of the relative LIF signal thus gives the absolute rate constant: this has been used to determine the absolute concentration of the $\mathrm{N}_{2}^{+}$ion in the bulk of a parallel plate reactor as $1 \times 10^{10} \mathrm{~cm}^{-3}$ at a molecular nitrogen concentration of $6.5 \times 10^{14} \mathrm{~cm}^{-3}[14]$.

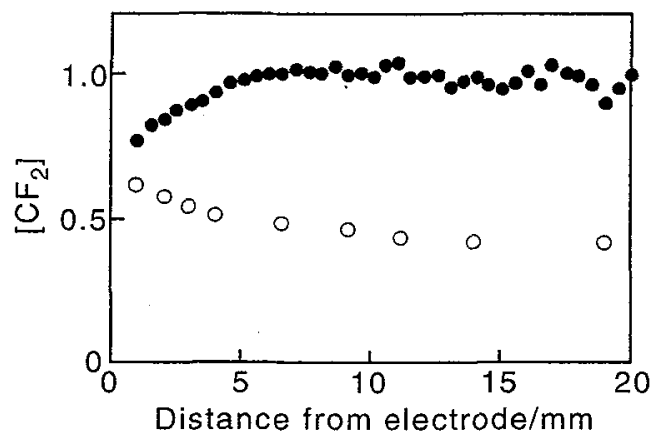

Figure 1: $\mathrm{CF}_{2}$ radical signals observed by LIF as a function of height above the RF driven electrode in a parallel plate reactor. Two separate sets of results are shown for a plasma in $\mathrm{CF}_{4}$ (filled circles) and an equimolar mixture of $\mathrm{CHF}_{3} / \mathrm{Ar}$ (open circles), both at a total pressure of $50 \mathrm{~m}$ Torr and the same applied power. As can be seen, the LIF signals behave very differently close to the electrode. The radical concentrations have been put on an absolute basis as explained in the text: for the data at $20 \mathrm{~mm}$ the concentrations in $\mathrm{CF}_{4}$ and $\mathrm{CHF}_{3} / \mathrm{Ar}$ are $1 \times 10^{13}$ and $1.4 \times 10^{13} \mathrm{~cm}^{-3}$ respectively. Reproduced with permission from [6].

Figure 2 shows how the concentration of the $\mathrm{N}_{2}{ }^{+}$ion varies with height above the driven electrode in a parallel plate reactor. The plasma sheath in this case is observed visually to extend about $12.5 \mathrm{~mm}$ above the electrode surface, and it can be seen that as the surface is approached and the ions are accelerated to it, their concentration drops as a consequence of their increased velocities. The limiting concentration that we have been able to measure in these experiments is about $2 \times 10^{8} \mathrm{~cm}^{-3}$, and we now consider whether or not this number can be considered a "reasonable" detectivity limit for LIF in plasmas. 


\subsection{Detectivity}

In principle, it is possible to detect a single atom or ion by LIF. With a reasonably intense continuous-wave laser it is possible to saturate the absorption transition resulting in a maximum spontaneous fluorescence rate whilst the laser is active of half a photon per upper level radiative lifetime. Taking the example of atomic caesium, excitation by a laser diode at $852 \mathrm{~nm}$ will saturate at an intensity of $65 \mu \mathrm{W} \cdot \mathrm{mm}^{-2}$, and fluorescence photons will be emitted at a rate of 16 million $\mathrm{s}^{-1}$. The emission from thermal population of the upper level will in comparison be negligible. By measuring the change in detected fluorescence signal in phase with wavelength modulation of the laser on and off the resonance wavelength, the background emission and laser scatter are rejected.

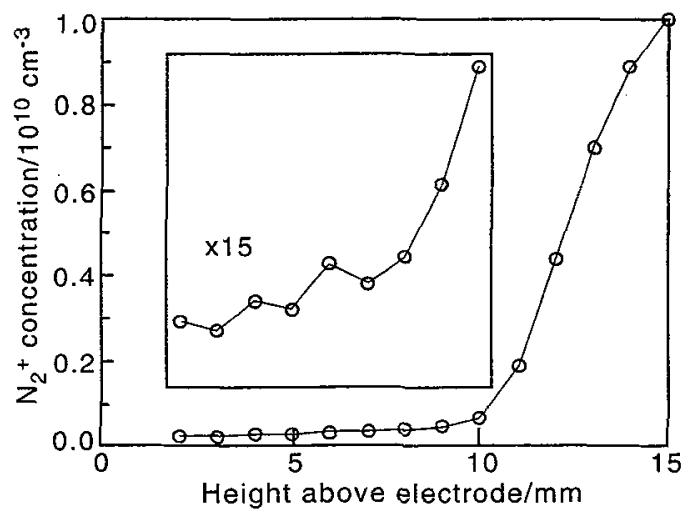

Figure 2: Measurements of the variation of $\mathrm{N}_{2}^{+}$ion concentrations as a function of height above the driven electrode in an $\mathrm{RF}$ capacitatively coupled parallel plate reactor. LIF signals were put onto an absolute basis by measurements of the $\mathrm{N}_{2}^{+}$decay rates under conditions where this was dominated by dissociative recombination. The smallest concentrations measured were approximately $2 \times 10^{8}$ ions $\mathrm{cm}^{-3}$. Total pressure 20 mTorr. Reprinted with permission from J. Appl. Phys. 81 (1997) 5945 . (O 1997 American Institute of Physics.

Our first estimate of the detectivity limit for species in a plasma is thus one atom, ion or molecule in the appropriate electronic, vibrational and rotational state within the observed volume. Taking a required spatial resolution of $1 \mathrm{~mm}^{3}$ and assuming that we are dealing with a molecular species with $1 \%$ of the population in the required initial state, we arrive at a density of $10^{5} \mathrm{~cm}^{-3}$, although the signal will at such concentrations vary as the single species diffuse into and out of the observed volume. Of course, the efficiency of detection of the fluorescent photons will not be unity - typically, because of the solid angle subtended by the detector and the detector efficiency, we shall collect less than $1 \%$ of the emitted light, even with F/2.8 optics. If we increase the species concentration to achieve once again the full single-atom count rate, and thus also reduce the statistical signal variation, we arrive at a concentration of the order of $10^{8} \mathrm{~cm}^{-3}$, which proves in practice to be a good rule of thumb.

For pulsed laser excitation, the pulse length is generally of the order of or less than the radiative lifetime, and thus the saturated spontaneous fluorescence signal will not exceed one photon per atom or ion per laser pulse. Single photon detection is nonetheless possible, and the above logic thus continues to apply: Goeckner et al [15] have measured argon ions by pulsed laser-induced fluorescence with a precision of the order of $10^{7} \mathrm{~cm}^{-3}$, whilst for molecular species some of our own results are shown in fig 2 at a total concentration of $2 \times 10^{8} \mathrm{~cm}^{-3}[14]$, and we shall see later that it is possible to achieve some degree of velocity resolution at this level. Both cases are typical of ion densities in plasma sheaths. The low duty cycle of a pulsed laser however requires a longer averaging time than for c.w. experiments: Sadeghi et al [16] have compared c.w. and pulsed LIF, and found an immediate improvement in signal-to-noise ratio upon changing to the c.w. system, even though the species sensitivity was reduced as a result of improved Doppler velocity resolution. 
Signal fluctuations are not, unfortunately, the sole sources of noise in LIF measurements of plasma species, for the plasma - unlike single trapped ions, for example - radiates light of its own accord. This plasma emission, due principally to electron impact excitation of the observed species, occurs at a much lower level than that due to saturated LIF, but a typical imaging system will be sensitive to plasma emission at all points within the field of view. The effective contributing volume may be several orders of magnitude larger than that observed by LIF; it may also correspond to regions of higher species concentration, for example if the plasma sheath is observed through, or partly through, the plasma bulk. Plasma emission which occurs at wavelengths other than that at which LIF is observed may be removed by suitable filters placed before the detector. That which occurs at the observed wavelength is then eliminated from the signal by phase-sensitive measurement as the laser is switched or tuned on and off resonance, although it will continue to add to the measurement noise.

There are, in addition, two routes through which the laser may contribute to the observed signal: scatter of the laser beam by the plasma and plasma chamber, and scatter of fluorescence induced outside the observed region. Direct laser scatter may be attenuated by the detector filters if we are observing offresonance, but potentially has a much larger initial intensity: care should therefore be taken to eliminate scattering objects from the line of sight, and to reduce their reflectivity wherever possible. Scattered LIF cannot be so removed, although it should be attenuated by the scattering process itself. Nonetheless, if we again consider LIF measurements of sheath species probed by a laser beam entering through the plasma bulk, it is clear that the longer path length through the bulk and the higher species density there will result in a far larger yield than from the small and rarefied region being probed. If the laser beam runs along the axis of the plasma chamber, then the cylindrical walls will reflect some of the emission onto the axial region of observation. If it is then scattered, it will be indistinguishable from true LIF unless careful diagnostic tests are carried out.

Sensitive LIF experiments will therefore use carefully designed imaging to collect the fluorescence without accepting too much background plasma emission or laser scatter, and the light will be passed through filters to remove both plasma emission and laser wavelengths. Wavelength modulation allows direct laser scatter to be eliminated, and background emission may be rejected by either wavelength or intensity modulation of the laser. Finally, comparison with the signal when the plasma has been extinguished will often confirm that the observed signal is indeed laser-induced fluorescence from the species and region of interest.

\section{ENERGIES}

LIF is a quantum state resolved measurement, and thus can be used to determine the distribution of the species over the populated quantum states. If the species are in Boltzmann equilibrium then this distribution will correspond to a temperature, but we should note that there is no a priori reason to assume that this will be true in a plasma. For a temperature to be established within a given degree of freedom we need the rate of formation of a specific quantum state to be slow in comparison with the rates of the energy transfer processes within that degree of freedom. The latter processes may take place in 10-100 collisions for equilibration within translational and rotational degrees of freedom, but may require many more than this for vibrational energy transfer. Furthermore, although different degrees of freedom may be characterised by temperatures they need not necessarily be the same. This will be particularly true for vibrational distributions in comparison with those for rotational and translational degrees of freedom owing to the relatively slow rates of energy transfer out of vibration into these modes.

Figure 3 shows part of what is known as a fluorescence excitation spectrum of the $\mathrm{N}_{2}^{+}$ion near $400 \mathrm{~nm}$, in which the total fluorescence is gathered as the laser wavelength is swept. The main structure can be identified as lines in the $\mathrm{R}$ branch of the $\mathrm{B}^{2} \Sigma^{+}-\mathrm{X}^{2} \Sigma^{+}(0,0)$ band, and the intensities of these can be converted into relative populations if the following factors are known or understood. First, a knowledge of the line strengths (equivalent to the absorption cross sections) is needed - these are straightforwardly calculable. Secondly, the excitation process is not isotropic in the laboratory frame, as the laser has a propagation vector and is normally linearly polarised. Even for an isotropic samiple (by isotropic here we mean a random 
distribution of the transition dipoles of the absorbing species) this means that the fluorescence itself is not spatially isotropic and is often polarised. Corrections should be applied to take this into account, and a "user friendly" guide to the extraction of populations under these conditions [17] has been extensively used in the fields of photodissociation and reaction dynamics where the distribution of transition dipoles probed by LIF can be markedly non-isotropic. In plasmas the distributions are assumed to be isotropic, and the polarisation corrections assumed to be small. Neither is necessarily true: energy transfer processes involving drifting ions have been shown to lead to molecular frame alignment [18], and polarisation corrections can be of the order of $\pm 10 \%$ if transitions from different branches or from quantum states with low values of $J$ are measured. For neutral species these effects however are generally small. Thirdly, saturation can again be a problem, and we consider its effect later on in this section.

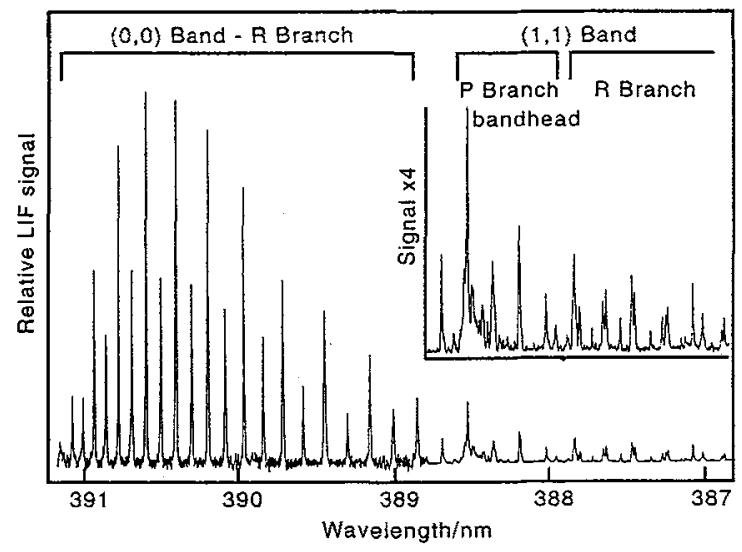

Figure 3: Laser excitation spectrum between 387 and $391 \mathrm{~nm}$ of the $\mathrm{N}_{2}^{+}$ion excited in the $(0,0)$ band of the $\mathrm{B}^{2} \Sigma^{+}-\mathrm{X}^{2} \Sigma^{+}$transition in the bulk of a plasma of nitrogen at $50 \mathrm{~m}$ Torr. The $\mathrm{R}$ branch of the $(0,0)$ band can be analysed to reveal the rotational distribution of the ground state ions, which in this case corresponds to a Boltzmann temperature of $355 \mathrm{~K}$. At the low wavelength end of the spectrum part of the $(1,1)$ band can be seen, and this allows an estimate of the relative vibrational populations of the $v=0$ and 1 levels to be made.

An analysis of the data in Figure 3 shows that in the plasma bulk the $\mathrm{N}_{2}{ }^{+}$ions have a distribution which corresponds closely to a temperature, in this case $355 \pm 15 \mathrm{~K}$. This is not surprising: rotational equilibration in this ion is expected to be rapid under the experimental conditions ( $50 \mathrm{~m}$ Torr total pressure in this case), and we would expect this degree of freedom to be equilibrated with the local translational temperature. We note that in Figure 3 at shorter wavelengths, signal is seen in the $(1,1)$ band of the transition, and this allows us to estimate the relative vibrational populations of $v=0: v=1$ as $1: 0.07$. If this corresponded to a temperature (we cannot confirm this, as our detectivity precludes observations of higher vibrational levels) it would be around $1200 \mathrm{~K}$, showing the marked difference between the rotational and vibrational degrees of freedom. Vibrational distributions are little studied in plasmas, and the importance of such excited species warrants investigation. For example, the electron impact cross section for a dissociation or ionisation process may be considerably enhanced by vibration, owing to a better overlap of geometries between the vibrationally excited state and the electronically excited state which leads to fragmentation(the electron impact equivalent of a Franck-Condon factor in photon absorption). The process requires electrons of lower energy than for the ground state, another factor which would enhance the rate of the excitation process. Further investigation of such effects is required.

Figure 4 shows a high resolution scan of the $\mathrm{R}(6)$ line of the $(0,0)$ band of $\mathrm{N}_{2}{ }^{+}$, taken in the plasma bulk with the laser beam propagating parallel to the electrodes (and thus perpendicular to any DC fields in the chamber). The double-peaked structure is caused by the spin rotation doubling in the ${ }^{2} \Sigma^{+}$states of the transition, and the widths are the result of the speed distribution of the ions in the laser propagation direction. The fit is that which would be expected if the translational distribution corresponded to the same 
temperature, within experimental error, as that of the rotational distribution. We note that this is not a very precise measurement of temperature, as the Doppler width scales as $T^{1 / 2}$. We note also that ions are affected by fields, and we expect this to be manifested in the speed distributions perpendicular to the electrodes. Within the plasma bulk we find the velocity distribution to be isotropic (and well represented by a temperature) within our experimental precision, but as the ions are driven through the sheath their speeds in this perpendicular direction increase. Figure 5 shows data as a function of height above the driven electrode: fast ions are seen, increasing in importance as the electrode is approached. These fast ions are always accompanied by an unexpected component of slow ions, the origin of which is still unclear but which may have been from ionisation by secondary electrons emitted from the electrode surface [14]. The non-intrusive nature of the laser beam means here that the velocity distributions can be measured throughout the plasma, and because the technique measures concentrations of particles as well as their speeds, particle fluxes can be estimated throughout the range of particle energies.

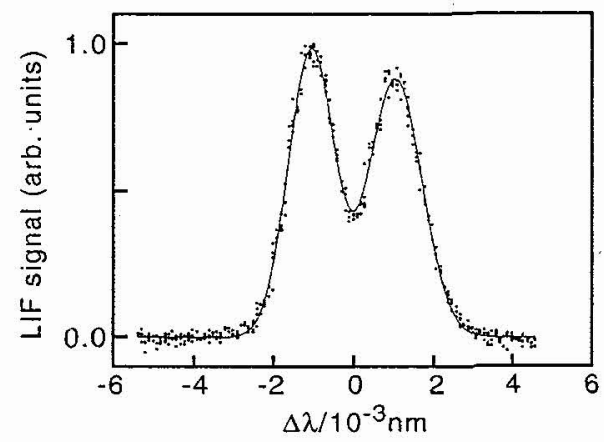

Figure 4: High resolution LIF scan over the $\mathrm{R}(6)$ line of the $\mathrm{N}_{2}{ }^{+}$band shown in Figure 3. The two peaks result from spin doubling of the rotational levels in the ${ }^{2} \Sigma^{+}$states, and the fit is that which would be expected from a Boltzmann distribution of the ions velocities at the same temperature as for the rotational distribution of Figure 3.
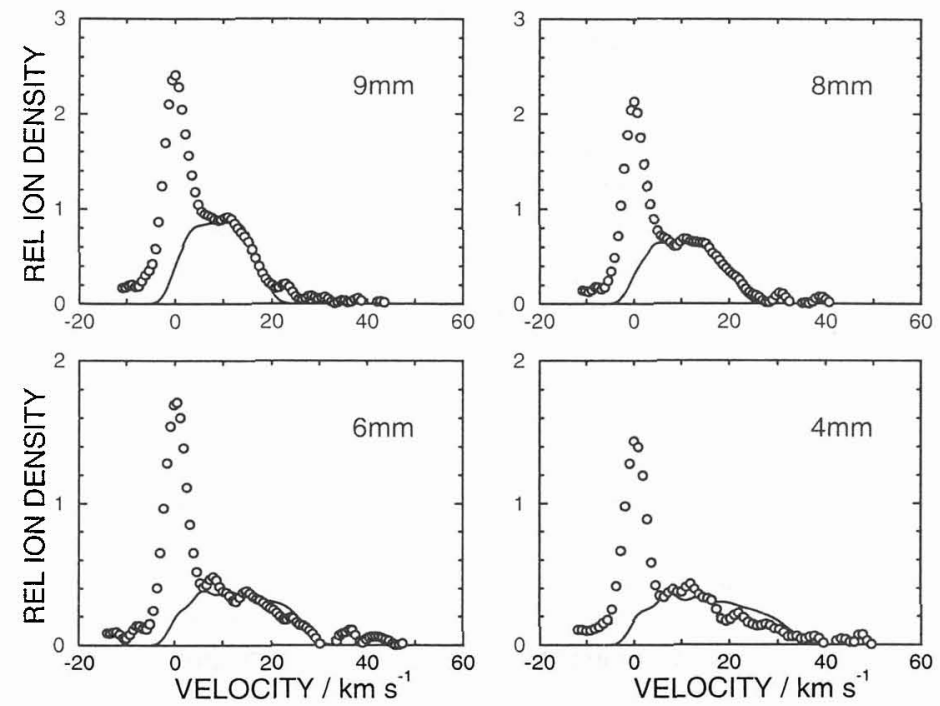

Figure 5: Measured relative $\mathrm{N}_{2}{ }^{+}$ion densities as a function of velocity component perpendicular to the electrode surface at distances of $9,8,6$ and $4 \mathrm{~mm}$ above the driven electrode and at a pressure of $20 \mathrm{~m}$ Torr. The total ion concentrations are respectively $4.6,3.9,3.0$ and $2.6 \times 10^{8} \mathrm{~cm}^{-3}$ and the curves are plotted in the same relative units. The solid curves are theoretical predictions, which, whilst reproducing well the velocity distributions of the fast ions, are unable to account for the persistent slow component. The latter may be caused by ionisation from fast electrons emitted from the driven electrode. Reprinted with permission from J. Appl. Phys. 81 (1997) 5945. (C) 1997 American Institute of Physics. 


\subsection{Pitfalls of saturation}

At low laser intensities, the processes of laser-induced excitation and subsequent fluorescence are simply related: for each laser photon absorbed an atom, ion or molecule is excited, and there is a constant probability that the subsequent decay will be via spontaneous radiation of a fluorescence photon, the detectability of which will depend upon the solid angle subtended by the detector and any loss processes or inefficiencies therein. Provided that the probabilities and loss mechanisms are known and that the sample is optically thin, the ratio of fluorescent signal to laser intensity will hence give the absolute species concentration; if the sample is not optically thin then attenuation of the exciting laser beam and reabsorption of the fluorescence must of course be taken into account. Such is the basis for measurement of absolute species concentrations by LIF. The calibration parameters may be established experimentally in a number of ways, as has been discussed in Section 2 .

When the same principle is used to determine the vibrational and rotational distributions, it must be noted that the fluorescence yield depends upon rotational and vibrational quantum number as well as electronic state. The vibrational dependence is characterised by the Franck-Condon factor $\mathrm{F}\left(v_{1}, v_{2}\right)$, which simply depends upon the overlap of the initial and final vibrational wavefunctions. The rotational dependence of the fluorescence yield is described similarly by the Hönl-London factor $S\left(J_{1}, J_{2}\right)$, which accounts for the initial and final state angular momenta $J_{1,2}$ and the requirement that this be conserved $\left(\Delta J=J_{1}-J_{2}=0, \pm 1\right)$. The total absorption rate $B_{12}$ (per unit spectral radiation density) from state 1 to state 2 is thus proportional to the product

$$
\mathrm{B}_{12} \propto \mathrm{R}_{12} \mathrm{~F}\left(v_{1}, v_{2}\right) \frac{\mathrm{S}\left(\mathrm{J}_{1}, \mathrm{~J}_{2}\right)}{g_{1}}
$$

where $R_{12}$ is the square of the dipole matrix element between the two electronic states and $g_{1}$ the initial state degeneracy (here the conventional notation is taken that the $S\left(J_{1}, J_{2}\right)$ sum over all $J_{2}$ to $\left.g_{i}\right)$. If the rotational distribution is required - it is often a good measure of the species translational temperature - then measurement is often made at high $J_{1}, J_{2}$ when the factor $S\left(J_{1}, J_{2}\right) / g_{1}$ loses its $J$ dependence. This is not however always possible, and with diatomic hydrides, for example, the rotational level spacing may be large enough that only the low $J$ states are thermally populated. In such cases, correction for the Hönl-London factors will be essential.

At high laser intensities typical of pulsed excitation, saturation occurs: the rate of excitation is sufficient that the ground (or lower) state population is depleted by the laser excitation before the end of the laser pulse or, in the continuous-wave case, before the atoms have had a chance to decay. The excitation rate is thus reduced whilst the converse process of stimulated emission competes with spontaneous fluorescence, and the signal becomes a sub-linear function of laser intensity. Ultimately, the strong laser field induces Rabi oscillations of population between the upper and lower levels: the mean excitation probability will be a half, and the fluorescence rate (if radiative decay is dominant) will be constant at half a photon per atom per radiative lifetime. Such saturation is, in principle, reproducible, and may therefore be calibrated, but the nonlinearity means that care must be taken if fluctuating signals are averaged, and in general it is necessary that the laser be stable for reliable results to be obtained.

\subsubsection{Saturation of homogeneouslybroadened species by monochromatic illumination}

For monochromatic illumination (laser linewidth $<\mathrm{A}_{12}$ ) of a homogeneously broadened sample of atoms, ions or molecules, by a laser of intensity $I(v)$ and frequency $v$, the fraction of the population in the excited state will be given simply by $[19,20]$

$$
\frac{-\sigma_{12}(v) \beta}{\left(1+g_{1} / g_{2}\right) h v} \frac{I(v)}{1+I(v) / I_{S}} .
$$


$I_{S}$ is the saturation intensity for the species, given by

$$
I_{s}(v)=\frac{h v A_{12}}{\sigma_{12}(v) \beta}
$$

where $A_{12}$ is Einstein coefficient of spontaneous emission between the final and initial states, $\sigma_{12}(v)$ is the absorption cross-section for the transition and $\beta=\tau_{2} A_{12}\left[1+\left(1-\tau_{2} A_{12}\right) g_{2} \tau_{1} / g_{1} \tau_{2}\right]$ where $\tau_{1,2}$ and $g_{1,2}$ are respectively the lifetimes and degeneracies of the initial and final states. With fully-allowed atomic transitions, the saturation intensity can be a few tens or hundreds of watts per $\mathrm{m}^{2}$, easily reached by c.w. lasers. Note that the saturation intensity depends, through $\beta$, upon the upper and lower level lifetimes, which are reduced at pressures high enough to broaden the natural atom or ion linewidth: the LIF yield, as well as the saturation characteristics, may thus be functions of the plasma pressure and other parameters which affect the population and quenching rates.

Vibrational and rotational structure typically raises the saturation intensity due to the Franck-Condon and Hönl-London factors by an order of magnitude or more, but saturation nonetheless remains possible with pulsed dye lasers, even when of broad bandwidth. Saturation has a somewhat different meaning with pulsed lasers, as we shall see in Section 3.1.3. First, however, we consider the effect of inhomogeneities in the laser-species interaction, and show that a linear dependence of LIF signal upon laser intensity by no means implies that saturation may be neglected.

\subsubsection{Saturation of inhomogeneouslybroadened species}

The fluorescence intensity from a homogeneously-broadenedsample (i.e. indistinguishable species) will rise with the laser intensity at low levels to a shoulder at $\mathrm{I}_{\mathrm{S}}$, and approach a constant fluorescence intensity thereafter. The situation is not, however, so simple when the sample is inhomogeneously broadened - i.e. the absorption lines of the plasma species are distributed due to thermal Doppler shifts, fine spectroscopic structure or spatial variations in field-induced Stark or Zeeman shifts. In such circumstances, different classes of species will be saturated to different extents for a given laser wavelength. In the case of a Dopplerbroadened sample, for example, the fluorescence signal will continue to increase with laser intensity even once the on-resonance species are strongly saturated: the signal essentially depends upon the proportion of species for which the process is saturated, which proves to vary as $I /\left(1+\mathrm{I} / \mathrm{I}_{\mathrm{S}}\right)^{1 / 2}[20]$.

Inhomogeneities in the interaction between the plasma species and the laser may also be due to spatial variations in the laser intensity, so that saturation is achieved at the centre of the laser beam before it occurs at the edges. In such cases, the fluorescence signal will increase with laser intensity even if the plasma species are homogeneously broadened [21].

It should thus be clear that a linear dependence of fluorescence upon laser intensity will not necessarily indicate the absence of saturation.

\subsubsection{Saturation by non-monochromaticillumination: laser linewidth $>A_{21}$}

For the general case of broad-band c.w. laser excitation, it is necessary to integrate equation (2) across the laser emission spectrum:

$$
\frac{\beta}{\left(1+g_{1} / g_{2}\right) h v} \int \sigma_{12}(v) \frac{\mathrm{I}(v)}{1+\mathrm{I}(v) / I_{S}} \mathrm{~d} v
$$

where $I(v)$ is the laser emission spectrum. The absorption cross-section $\sigma_{12}(v)$ contains the spectral sensitivity of the species, and a given species class thus sees only that part of the laser intensity which lies within its homogeneous linewidth. For specific cases, a saturation intensity may thus be calculated, and it is 
clear that an intense laser may be sufficiently broad in bandwidth that it avoids saturating the species because the spectral intensity is low.

For pulsed laser excitation with a duration shorter than the excited state lifetime, saturation of the fluorescence signal will not occur until intensities rather higher than those indicated above, which correspond to significant excitation within an atomic lifetime rather than the shorter laser pulse duration: the $A_{12}$ term should be replaced by the reciprocal of the pulse length. In such cases, account must also be taken of the laser bandwidth which will, by virtue of the finite pulse duration alone, be broader than the atomic absorption lineshape; the saturation intensity thus becomes proportional to the inverse square of the pulse length. If relaxation and spontaneous emission during the laser pulse duration $\tau_{\text {pulse }}$ may be neglected, then we may define a pulsed saturation intensity - at which the initial state population becomes significantly depleted - by

$$
\mathrm{I}_{S}=\frac{\mathrm{h} v \tau_{12}}{\sigma_{12} \tau_{\text {pulse }}^{2}}
$$

where $\tau_{2}$ is the upper level lifetime. For the $308.0 \mathrm{~nm} \mathrm{Q}_{1}(2)$ transition between the $\mathrm{A}^{2} \Sigma^{+}(\nu=0)$ and $\mathrm{X}^{2} \Pi(v=0)$ states of the $\mathrm{OH}$ radical, excited by a dye laser pulse of $10 \mathrm{~ns}$ duration, the saturation intensity will be $700 \mathrm{~kW} . \mathrm{cm}^{-2}$, corresponding to a fluence of $70 \mu \mathrm{J}_{\mathrm{mm}}{ }^{-2}$, easily achieved by the output of a commercial pulsed dye laser. This transition is not exceptionally strong; the upper state radiative lifetime is $700 \mathrm{~ns}$, and in species such as $\mathrm{CF}, \mathrm{NO}$ or $\mathrm{CF}_{2}$ saturation will occur at lower fluence levels. The above expression assumes, of course, that the laser bandwidth is entirely due to the finite pulse duration: this is a reasonable approximation for many lasers, whose single-pulse spectra are dominated by one or two longitudinal modes.

Modification of equation (4) for pulsed lasers is in many cases of only academic interest. Broad-band pulsed lasers owe their spectral distributions to shot-to-shot jitter and multiple longitudinal (and transverse) resonator modes. The above expression is valid for a single-pulse laser spectrum, across which there is assumed to be no phase coherence, and in general the difficulty of obtaining appropriate data and performing such integrations will not be sufficiently worthwhile. For the case of a constant and uniform intensity Gaussian lineshape, the significance of saturation for LIF measurements in Doppler-broadened plasma species has been carefully considered by Goeckner and Goree [22]; the reader should nonetheless be aware that for many lasers the approximation to an incoherent Gaussian may be very poor.

\subsubsection{Dealing with saturation in laser-inducedfluorescence}

It is apparent that at low intensities, the fluorescence yield is directly proportional to the product of laser intensity and species concentration, with a proportionality constant which may be derived either theoretically or by calibration, as discussed in Section 2. In such circumstances, the use of LIF to determine absolute species concentrations is straightforward. If the sample species are inhomogeneously broadened, then sweeping the laser wavelength gives the relative populations of the corresponding classes, enabling velocity distributions and so on to be determined. It is clear, nonetheless, that the exciting laser must be narrow or at least reproducible in bandwidth: if the laser bandwidth is broad, the effective laser intensity is only that fraction which lies within the homogeneous linewidth of the observed species.

If the laser spectral intensity is sufficient to saturate any of the species, then considerably more care is required in the interpretation of laser-induced fluorescence measurements, for the laser lineshape and the inhomogeneity of the laser-species interaction play crucial rôles in determining the fluorescence yield. Provided that the laser parameters are stable, a pragmatic calibration is nonetheless possible, as has been shown by Engelhard [21]; Cunge [10] meanwhile has demonstrated a neat method of cross-referencing the LIF yields from two different species by scaling according to the saturation parameter. It is also possible to compare the relative yields of two transitions of differing cross-section which originate from the same quantum level, such as the main and sub-branch transitions of the $\mathrm{OH}$ molecule [23]. 
The pitfalls of saturation in laser-induced fluorescence are two-fold. Firstly, saturation may go unrecognised because the fluorescence signal intensity continues to show a roughly linear dependence upon laser intensity: if a low-intensity calibration is applied to such data, the species concentration will be underestimated. Secondly, if the relation between laser and signal intensities is non-linear, then the average of a jittering or noisy signal may reflect the variation in laser intensity as well as the mean, and true calibration will in such circumstances be complex. The practitioner of LIF needs to be fully aware of these problems.

\section{TEMPORAL BEHAVIOUR}

Modulation of the power source applied to a plasma clearly will result in a change of the rate of formation of the active species produced as a result of electron impact processes. The steady-state equilibrium concentration of these species depends upon the balance of the formation and loss rates, and if the time scale of the modulation is slower than that on which the equilibrium is re-established, then the time history of the reactive species can be followed with ease and kinetic information extracted. Section 2 above demonstrates how $100 \%$ modulation of a $\mathrm{N}_{2}$ plasma can lead to information on the concentration of the dominant $\mathrm{N}_{2}{ }^{+}$ion when the loss process is dissociative recombination. One of the most useful pieces of information that can be derived from experiments on radicals in plasmas concerns the loss rates that can be measured on surfaces processes which are often the dominant removal steps in plasmas and are significant in technologically important processes such as plasma etching, polymerisation and ion implantation.

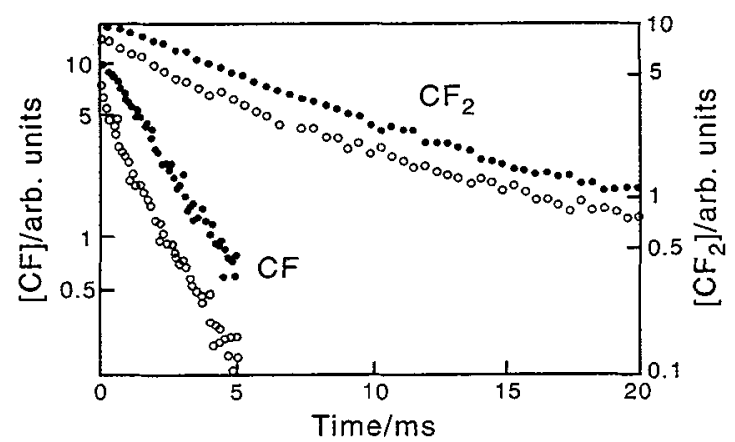

Figure 6: Semi-logarithmic plots of the variation in concentration of the $C_{2}$ and $C F$ radicals, observed by $L I F$, after the extinction of a plasma of $50 \mathrm{mTorr} \mathrm{CF}_{4}$. Closed and open circles represent the decay of the species measured at two different locations, 20 and $2 \mathrm{~mm}$ above the driven electrode respectively. Under these conditions the decays are seen to be reasonably well represented by exponential loss processes, with that for CF being markedly faster than for $\mathrm{CF}_{2}$. Reprinted with permission from $J$. Appl. Phys. 66 (1989) 5251. (O 1989 American Institute of Physics.

We give an example of the decay characteristics of radicals probed in a cylindrically symmetric RF excited parallel plate reactor [7] when the excitation source is removed. Under some conditions the resultant decay is well represented by a single exponential, and an example for both $\mathrm{CF}$ and $\mathrm{CF}_{2}$ radicals in a $\mathrm{CF}_{4}$ plasma is given in Figure 6. The time history of the radicals contains contributions from diffusion processes, losses in the gas phase by chemical reaction, and losses to the walls, and under some conditions the contribution from the wall loss process can be put on a quantitative basis. What can be estimated is a "phenomenological loss coefficient" [24], defined as the fraction of the particle flux to the surface which does not return to the gas phase on the time scale of the experiment. In Figure 1 we noted that radicals can have a marked spatial distribution within a reactor, and that this reflected formation and loss at the surfaces. For a full understanding of these processes both the spatial and temporal behaviour are required [8], and the complex behaviour that can occur is illustrated in Figure 7 where the spatially dependent loss phenomena of the $\mathrm{CF}_{2}$ radical in a $\mathrm{CHF}_{3} / \mathrm{Ar}$ plasma are shown. Plasma extinction leads to an initial rise of the concentration in the plasma bulk ( $20 \mathrm{~mm}$ from the electrode surface) because of diffusion from the higher 
concentrations of species present in the steady state near the electrode (illustrated in Figure 1). On re-ignition this concentration drops markedly, suggesting a homogeneous loss process involving other species formed in the plasma.

These results illustrate the type of information that can be found from such time dependent measurements, but we finish this section with two caveats. The first is that the determination of the loss coefficient tells us nothing about the chemical fate of the species lost on the surface. Some information can be obtained by looking for product molecules, for example the $\mathrm{SiF}_{2}$ radical formed by $\mathrm{F}$ atom etching of $\mathrm{Si}$ substrates [25], and observable by LIF near $225 \mathrm{~nm}$. The second more general point concerns LIF detection, and is illustrated by observations of ground state $O\left({ }^{3} \mathrm{P}\right)$ atoms, for which a convenient detection scheme is two photon excitation at $226 \mathrm{~nm}$ which populates the $3 \mathrm{p}{ }^{3} \mathrm{P}$ level and is followed by fluorescence to the lower $3 \mathrm{~s}^{3} \mathrm{~S}$ level at $845 \mathrm{~nm}$. Dye lasers can produce considerable output at $226 \mathrm{~nm}$, and this is enough to create a population inversion between these fluorescing levels, which then can result in amplified stimulated emission (ASE) along the axis of the laser beam. The process is well understood, but can in some cases have an effect on the LIF diagnostic signal, as the ASE process will compete with spontaneous fluorescence which is thus reduced. This can be a problem when the depletion is a non-linear function of atom density, and an effect of this kind, causing a fluorescence reduction of the order of $30 \%$, has been reported [26]. Our own measurements of the decay rates of $\mathrm{O}$ atoms following plasma extinction have demonstrated that erroneous results can be obtained if ASE is strong, as the fluorescence depletion is non-linear in $\mathrm{O}$ atom concentration and hence in time [27]. The problem is solved (at the expense of signal) by using a lower laser power, and illustrates that care needs to be taken to ensure that the laser diagnostic is not itself adversely affecting the quantity being observed.

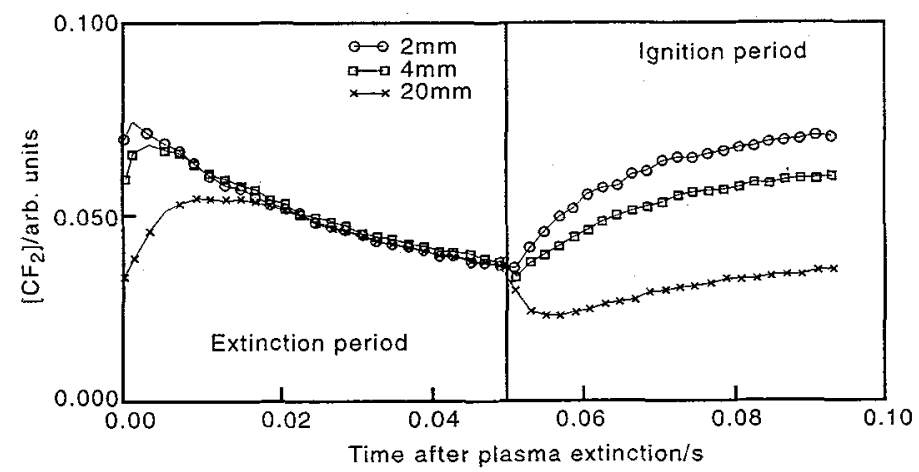

Figure 7: Variation of the time dependence of the LIF signal from $\mathrm{CF}_{2}$ with height above the driven electrode for a plasma of 200 mTorr equimolar $\mathrm{CHF}_{3} / \mathrm{Ar}$ as the power is switched off (extinction period) and on (ignition period). Close to the electrode the behaviour is similar to that seen in Figure 6, but in the bulk it is very different; an initial rise is seen following extinction and caused by diffusion of the higher concentration of radicals from close to the electrode. Re-ignition causes a noticeable fast drop in bulk concentration.

\section{FIELDS}

Charged particles react to fields, and a measurement of the field distribution in a plasma is crucial for understanding the methods by which energetic ions and electrons are formed, and the energies with which they collide with other species and with surfaces. LIF can do this in two ways. The first involves the Stark effect, in which energy levels are altered in the presence of an electric field. An example is given in Figure 8 from the work of Muraoka et al [28], in which LIF is observed following the excitation of the $11^{1} \mathrm{P}$ state of He from the metastable $2{ }^{1} \mathrm{~S}$ level in a DC glow discharge. Here advantage is taken of the fact that Stark splittings in He increase with principle quantum number $n$, and for the $n=11$ level the clear resolution in Figure 8 was obtained for an electric field of $90 \mathrm{~V} / \mathrm{mm}$, with the detection limit, determined simply by the laser bandwidth, being $21.5 \mathrm{~V} / \mathrm{mm}$. Excitation to higher $\mathrm{n}$ with a spectrally narrowed source improves this 
by a factor of 2 . More recently Stark shifts in atomic $H$ excited to $n=3$ by two-photon excitation of the ground state atom at $205 \mathrm{~nm}$ have yielded a sensitivity limit of $5 \mathrm{~V} / \mathrm{mm}$ [29].

The second method for LIF measurement of electric fields relies on the field mixing of levels of opposite parity in the upper electronic level [30]. The method is illustrated with reference to the best studied example, that of the $\mathrm{BCl}$ radical excited in its $\mathrm{A}^{\mathrm{l}} \Pi-\mathrm{X}^{\mathrm{l}} \Sigma$ transition, the $(0,0)$ band of which is at $272 \mathrm{~nm}$. The upper $A^{l} \Pi$ state has its rotational levels split into opposite parity components, depending upon whether the $\Pi$ electron is in an orbital which is symmetric or antisymmetric with respect to reflection through the plane of rotation ('lambda doubling'). In the absence of a field (and of collisions), excitation of for example an $R$ branch transition yields fluorescence only on $R$ and $P$ branch lines $(\Delta J= \pm 1)$, owing to the parity selection rules. Electric fields cause mixing of the states of opposite parity, and in the presence of a field a $Q$ $\operatorname{branch}(\Delta \mathrm{J}=0)$ is seen, the intensity of which relative to the fully allowed $\mathrm{R}$ and $\mathrm{P}$ branches gives a measure of the electric field. The field detectivity limits are of the same order as those measured by Stark splittings, and a useful list of the appropriate species for which this technique is possible has been published [28]. One of the most notable pieces of information that can be obtained through this technique is a measure of how the electric field changes with the phase of the applied voltage, for example during an RF cycle [31]. The limitation however is that the species needs to have a $\Sigma$ ground state, and this restricts the range of diatomic species that can be studied, eliminating for example radicals such as $\mathrm{OH}, \mathrm{CF}$ and $\mathrm{SiF}$ which have unpaired electrons but ${ }^{2} \Pi$ ground electronic states.

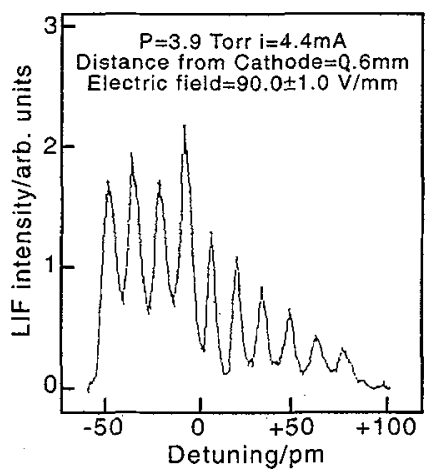

Figure 8: LIF spectrum near $321.25 \mathrm{~nm}$ of the He $11^{1} \mathrm{P} \leftarrow 2{ }^{1} \mathrm{~S}$ transition in a DC discharge, against laser detuning from centre wavelength. The peaks arise from the Stark splitting in the upper state, and from these data the DC field can be calculated. Reproduced with permission from [28]

\section{CONCLUSIONS}

Densities, spatial distributions, energies, fields and interaction kinetics of plasma species can all be measured in a non-invasive fashion by LIF: information crucial for the refinement of models low temperature plasmas. Yet lasers are only rarely used as diagnostics in the technological plasmas used for industrial processing, partly because lasers have hitherto been bulky, expensive and - in spite of their manufacturers' claims - in need of the attention of skilled operators.

The examples of laser-induced fluorescence measurements that we have given in this article all involve the use of laser radiation in the visible and u.v. spectral regions. These wavelengths can now be reached by efficient upconversion of the output of c.w. diode lasers manufactured in vast quantities for communications and optical reader systems. Efficient frequency doubling of c.w. radiation in $\mathrm{KNbO}_{3}$, for example, can yield several $\mathrm{mW}$ of light in the $430 \mathrm{~nm}$ region [32], and carefully designed resonators allow similar powers to be generated with few gaps from as low as $330 \mathrm{~nm}$ up to $500 \mathrm{~nm}$ and beyond. Even frequency quadrupling, which extends the range of these sources to below $250 \mathrm{~nm}$, has been reported. Such systems are already being considered for balloon-borne atmospheric measurements, where their small size, relatively low cost and ruggedness make laser-based diagnostics attractive. Diode lasers have an additional advantage over 
conventional devices in that their emission wavelength is directly controllable electrically, permitting extremely high stability to be maintained and allowing complex modulation and signal processing schemes to be tailored to the spectroscopic application.

Diode laser systems, only just emerging from the laboratory, may represent the future of in situ processing diagnostics, but their conventional cousins continue to be capable workhorses for plasma investigations, provided that the price and expertise can be afforded. A pulsed dye laser, pumped by an excimer or Nd:YAG source, or a continuous-wave system with the dye pumped by an argon ion or c.w. Nd:YAG laser, will put at its owners' disposal most of the techniques that we have described.

\section{References}

[1] The application of optical diagnustics to low pressure plasmas has been reviewed by Dreyfus R. W., Jasinski J. M., Walkup R. E., Selwyn G. S., Pure \& Appl. Chem. 57 (1985) 1265.

[2] Werle P., Mücke R. and Slemr F., Appl. Phys. B 57 (1993) 131; Güllïck T., Wagner H. E. and Slemr F., Rev. Sci. Instrum. 68 (1997) 230.

[3] Dorn H. P., Neuroth R. and Hofzumahaus A., J. Geophys. Res: 100 (1995) 7397.

[4] Childs M. A., Menningen K. L., Toyoda H., Anderson L. W. and Lawler J. E., Europhys. Lett. 25 (1994) 729.

[5] Hancock G. and Sucksmith J. P., J. Vac. Sci. Technol. A13 (1995) 2945.

[6] Hancock G., Lanyi L., Sucksmith J. P., and Woodcock B. K., Pure and Appl. Chem. 66 (1994) 1207.

[7] Booth J. P., Hancock G., Perry N. D. and Toogood M. J., J. Appl. Phys. 66 (1989) 5251.

[8] Booth J. P., to be published.

[9] Hancock G., Proc. Int. Symp. Laser-Aided Plasma Diagnostics 5 (1991) 254.

[10] Cunge G., Booth J. P., Derouard J., Chem. Phy.s Lett. 263 (1996) 645.

[11] Bischel W., Bamford D. and Jusinski L., Appl. Optics 25 (1986) 1215.

[12] Jacob W., Engelhard M., Möller W. and Koch A., Appl. Phys. Lett. 64 (1994) 971.

[13] Borysow J. and Phelps A. V., Phys. Rev. E 50 (1994) 1399; Visser R. J., Baggerman J. A. G., Poppelaars J. P. J. and Collart E. J. H., J. Appl. Phys. 71 (1992) 5792.

[14] Woodcock B. K., Busby J. R., Freegarde T. G. M. and Hancock G., J. Appl. Phys. 81 (1997) 5945.

[15] Goeckner M. J., Goree J., Sheridan T. E., Phys. Fluids B 4 (1992) 1663.

[16] Sadeghi N., Nakano T., Trevor D. J., Gottscho R. A., J. Appl. Phys. 70 (1991) 2552.

[17] Greene C. H. and Zare R. N., J. Chem. Phys. 78 (1983) 6741.

[18] Anthony E. B., Schade W., Bastian M. J., Bierbaum V. M. and Leone S. R., J. Chem. Phys. 106 (1997) 5413.

[19] Corney A. Atomic and Laser Spectroscopy (Oxford University Press, Oxford, 1977)

[20] Demtröder W., Laser Spectroscopy - basic concepts and instrumentation (Springer-Verlag, Berlin, 1996)

[21] Engelhard M., Jacob W., Möller W., Koch A. W., Appl. Opt. 34 (1995) 4542.

[22] Goeckner M. J., Goree J., J. Vac. Sci. Technol. A 7 (1989) 977.

[23] Brouard M., Lambert H. M., Short J. and Simons J. P., J. Phys. Chem. 99 (1995) 13571.

[24] Booth J. P. and Hancock G., Mat. Sci. Forum 140 (1993) 219.

[25] Vanhaelermeersch S., van Hoeymissen J., Vermeylen D. and Peeters J., J. Appl. Phys. 70 (1991) 3892.

[26] Tserepi A. D., Wurzberg E. and Miller T. A., Chem. Phys. Lett., 265 (1997) 297.

[27] Toogood M. J., D.Phil. thesis, Oxford University, 1991.

[28] Muraoka K. and Maeda M., Mat. Sci. Forum 140 (1993) 191.

[29] Booth J. P., J Derouard, Fadlalhah M., Cabaret L. and Pinard J., Opt. Commun. 132 (1996) 363.

[30] Moore C. A., Davis G. P. and Gottscho R. A., Phys. Rev. Lett. 52 (1984) 538. Mandich M. L., Gaebe C. E. and Gottscho R. A., J. Chem. Phys. 83 (1985) 3349.

[31] Yamagata Y., Miyama M., James B. W., Muraoka K., Maeda M. and Akazaki M., Proc. 10th Int. Symp. Plasma Chem. 2 (1991) paper 2.1-49.

[32] Lodahl P., Sorensen J. L. and Polzik E. S., Appl. Phys. B 64 (1997) 383. 